\title{
Cerebral ischemia at early postoperative period of direct revascularization for moyamoya disease: a case report and literature review
}

\author{
Xiao-Lin Chen ${ }^{1,2^{*}}$, Li Ma ${ }^{1,2,3^{*}}$, Yu Chen ${ }^{1,2,3^{*}}$, Jun-Lin Lu ${ }^{1,2,3}$, Xun Ye ${ }^{1,2,3}$, Rong Wang ${ }^{1,2,3}$, Yuan-Li Zhao \\ ${ }^{1}$ Department of Neurosurgery, Beijing Tiantan Hospital, Capital Medical University, Beijing 100050, China. \\ ${ }^{2}$ China National Clinical Research Center for Neurological Diseases, Beijing 100050, China. \\ ${ }^{3}$ Department of Neurosurgery, Peking University International Hospital, Peking University Health Science Center, Beijing 102206, China. \\ ${ }^{4}$ Center of Stroke, Beijing Institute for Brain Disorders, Beijing 100050, China. \\ ${ }^{5}$ Beijing Key Laboratory of Translational Medicine for Cerebrovascular Disease, Beijing 100050, China. \\ *Authors contributed equally.
}

Correspondence to: Dr. Yuan-Li Zhao, Department of Neurosurgery, Beijing Tiantan Hospital, Capital Medical University, No. 6 Tiantan Xili, Dongcheng District, Beijing 100050, China. E-mail: zhaoyuanli@126.com

How to cite this article: Chen XL, Ma L, Chen Y, Lu JL, Ye X, Wang R, Zhao YL. Cerebral ischemia at early postoperative period of direct revascularization for moyamoya disease: a case report and literature review. Neuroimmunol Neuroinflammation 2017;4:46-53.

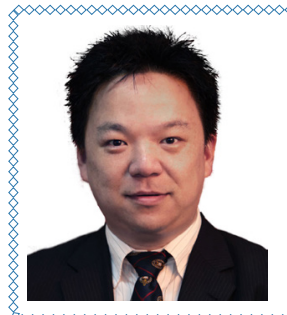

Dr. Yuan-Li Zhao is a Professor of Neurosurgery in the Department of Neurosurgery, Beijing Tiantan Hospital, Capital Medical University and China National Clinical Research Center for Neurological Diseases. He is also the Neurosurgeon-in-Chief and Chairman of the Department of Neurosurgery, Peking University International Hospital. He earned his medical degree from Peking Union Medical College in 1996. He was a visiting scholar at Massachusetts General Hospital, Johns Hopkins Hospital, Mayo Clinic and Barrow Neurological Institute. He is the Deputy President of the Young Neurosurgeon Committee of Chinese Neurosurgical Society and international member of American Association of Neurological Surgeons.

Article history:

Received: 12-01-2017

Accepted: 22-02-2017

Published: 24-03-2017

Key words:

Moyamoya disease,

cerebral revascularization, transient neurological events, perioperative management

\section{ABSTRACT}

Hypoperfusion and hyperperfusion could be causes of early postoperative complications that lead to neurological deterioration in patients with moyamoya diseases (MMD) after superficial temporal artery (STA) and middle cerebral artery (MCA) anastomosis. Here, the authors described a case of child-onset bilateral MMD that manifested transient cerebral ischemia in the contralateral hemisphere after left STA-MCA bypass in young adulthood. A new onset of cerebral ischemia in the contralateral hemisphere and transient neurological deterioration suggested the fragile hemodynamics of MMD during early perioperative period. Serial evaluation of postoperative cerebral hemodynamics and perfusion might facilitate targeted management in patients with unstable or advanced MMD.

cc) (i) (2) This is an open access article distributed under the terms of the Creative Commons Attributioncc) Non Commercial-ShareAlike 3.0 License, which allows others to remix, tweak, and build upon the work non-commercially, as long as the author is credited and the new creations are licensed under the identical terms. 


\section{INTRODUCTION}

Moyamoya disease (MMD) is one of the major causes of stroke in children and adults. It is characterized by progressive stenosis or occlusion of terminal portion of internal carotid arteries and development of fragile collateral vessels (moyamoya vessels). ${ }^{[1]}$ Revascularization surgery is a recommended therapy in these patients. ${ }^{[2]}$ Although the long-term outcome of direct revascularization through superficial temporal artery (STA)-middle cerebral artery (MCA) bypass is generally favorable, early postoperative neurological events are still frequently reported and contribute to neurological deterioration. ${ }^{[3]}$ The change of perioperative cerebral perfusion is suggested to be dynamic, therefore to identify the underlying mechanisms and risk factors might improve the postoperative management, especially in patients with advanced MMD and unstable hemodynamics. In MMD with unilateral STA-MCA bypass, symptomatic cerebral ischemia in the contralateral hemisphere occurs in about $3-14 \%$ of patients. ${ }^{[4-7]}$

We herein presented a case of childhood-onset bilateral MMD, which developed transient cerebral ischemia in the contralateral hemisphere after STAMCA bypass in her young adulthood. Transient weakness of the left extremities and acute cerebral infarctions in the contralateral hemisphere were observed from postoperative Day 1 to Day 6. The neurological deficit improved after intravenous infusion of fluid and free radical scavenger. There were no subsequent neurological events, and the preoperative neurological deficit significantly improved during the follow-up period. The mechanism and management of the neurological events in the early postoperative period were further discussed with a literature review.

\section{CASE REPORT}

A 21-year-old female with 5-year history of paroxysmal limbs weakness presented to our hospital with a reoccurrence of symptoms and slurred speech 15 days ago. There was no family history of MMD. Digital subtraction angiography (DSA) revealed extensive stenosis of the bilateral terminal portions of the internal carotid arteries (ICAs) and abundant moyamoya vessels on both sides, leading to a diagnosis of MMD. The bilateral anterior cerebral artery (ACA) and the right posterior cerebral artery (PCA) were not identified. The left PCA was also involved with stenosis and collaterals of vascular network. Collaterals from the posterior circulation formed between right occipital and parietal lobe [Figure 1]. Magnetic resonance imaging (MRI) showed multiple infarct lesions in the bilateral watershed. Perfusion computed tomography (CT) showed a reduced cerebral blood flow of the bilateral hemisphere with a mild domain on the left side [Figure 2].

Considering the patient had barylalia, a left STAMCA anastomosis was first performed between the parietal branch of the STA and MCA (M4 segment) supplying the frontal and parietal lobe. The intraoperative blood pressure (BP) remained between $120-140 \mathrm{mmHg}$. We followed our postoperative protocols with fluid infusion, a prophylactic anti-epileptic drug, aspirin, and statins. The patient did not display any neurological deficits after surgery until she appeared restless and delirious $8 \mathrm{~h}$ afterwards. We suspected hyperperfusion syndrome after revascularization, while the perfusion CT was not available at night. Consequently, the patient was managed with blood pressure control (systolic blood pressure between $110-130 \mathrm{mmHg}$ ) and intravenous fluid infusion. On the 1st day after surgery, she appeared to have barylalia and retrograde amnesia, thus intravenous edaravone (60 mg/day) was added. On the 2 nd day after surgery, the retrograde amnesia completely resolved, while left limb weakness appeared with muscle strength of Grade 3. Postoperative imaging on the 3rd and 6th day after surgery revealed an improved perfusion of left hemisphere, but identified a de novo lesion between the right temporal and occipital cortex with high intensity on $\mathrm{T} 2$ weighted image (T2WI) and diffusion weighted image (DWI) and decreased cerebral blood flow (CBF), which was in the contralateral hemisphere of STA-MCA anastomosis [Figure 3]. Fluid infusion ensuring euvolemia, and edaravone were continued with systolic blood pressure between $130-140 \mathrm{mmHg}$. The patient's left limb weakness was completely resolved by the 6th day after surgery. She was discharged 13 days after surgery with the barylalia relieved and no other neurological deficits during the latest follow-up period of 2 months.

\section{DISCUSSION}

Extracranial-intracranial direct anastomotic bypass is recognized as a treatment for ischemic MMD with favorable long-term outcome. ${ }^{[1]}$ In the acute postoperative period of STA-MCA bypass, however, transient neurological events or stroke can be observed despite the improvement of cerebral perfusion at the site of anastomosis. ${ }^{[1,3]}$ Rapid, increased local perfusion and perioperative hemodynamic fluctuations after bypass surgery might increase the risk of abnormal perfusion in the adjacent area or remote regions, especially in patients with advanced stage, bilateral, or unstable MMD. ${ }^{[8-10]}$ 
In MMD with unilateral STA-MCA bypass, symptomatic cerebral ischemia in the contralateral hemisphere more commonly occurs in patients with advanced stage (Suzuki stage 4 to 6), PCA involvement and postoperative hypotension on postoperative Day (POD) 1 and Day 2. ${ }^{[7]}$ Advanced Suzuki stage and PCA involvement indicate the progression of MMD. ${ }^{[11]}$ In these patients, cerebral hemodynamics might be unstable and more susceptible to cerebral infarction after fluctuations of perfusion during the perioperative period. ${ }^{[7]}$ The patient in this case had a bilateral advanced stage of MMD (stage 4 for the left and stage 3 for the right) with right PCA involvement [Figure 1]. Although the unilateral direct revascularization might directly influence the blood flow of the contralateral hemisphere through collaterals, the collaterals from the contralateral side, in the present case, were poorly developed and were mainly around the midline, which might not explain the ischemia of the remote convexity [Figure 1]. Retrospective analysis of DSA suggested that the right PCA collaterals to the watershed between parietal and occipital lobes were not well developed. Preoperative perfusion CT also indicated impaired perfusion in the aforementioned area [Figures 1 and 2]. These angioarchitecture and perfusion features might be the underlying factors contributing to the focal ischemic deterioration after stress, including surgery and delirium. Therefore, intensive perioperative care should be recommended for these MMD patients with high risk factors [Table 1]. ${ }^{[12]}$ Real-time monitoring of the cerebral blood flow (CBF) showed that the regional $\mathrm{CBF}$ after revascularization followed a variable pattern with significant decreases between $12 \mathrm{~h}$ and $24 \mathrm{~h}$ after surgery and at $36 \mathrm{~h}$ after surgery. ${ }^{[13]}$ This CBF pattern correlated with the onset of a transient neurological event in our case and suggested a careful blood pressure management during the early postoperative period. In addition, rather than arbitrarily maintaining the blood pressure between certain parameters, the blood pressure management should be referenced to the preoperative level to avoid a rapid decrease from the baseline.$^{[7]}$ Recent data suggested that prophylactic
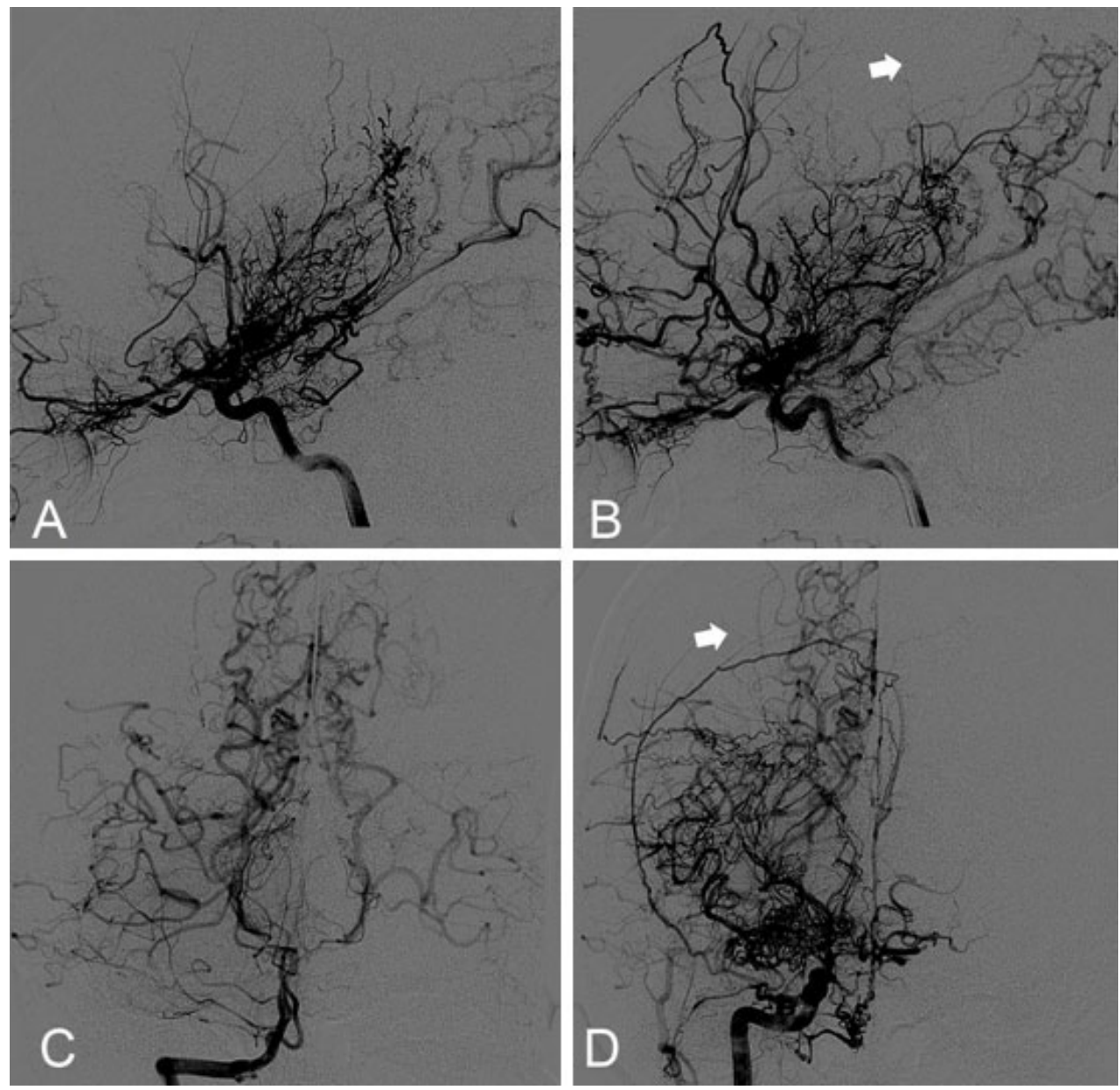

Figure 1: Preoperative angiography. Left ICA (A) and right ICA (B) angiography revealed a bilateral moyamoya disease. Vertebral angiography showed stenosis at proximal segment of right PCA and pooly developed left PCA, suggesting posterior circulation involvement (C). The anterior-posterior (B) and coronal (D) view of right ICA showed a watershed between parietal and occipital lobe, where there was lack of moyamoya collaterals from both anterior and posterior circulation (white arrow). ICA: internal carotid artery; PCA: posterior cerebral artery 


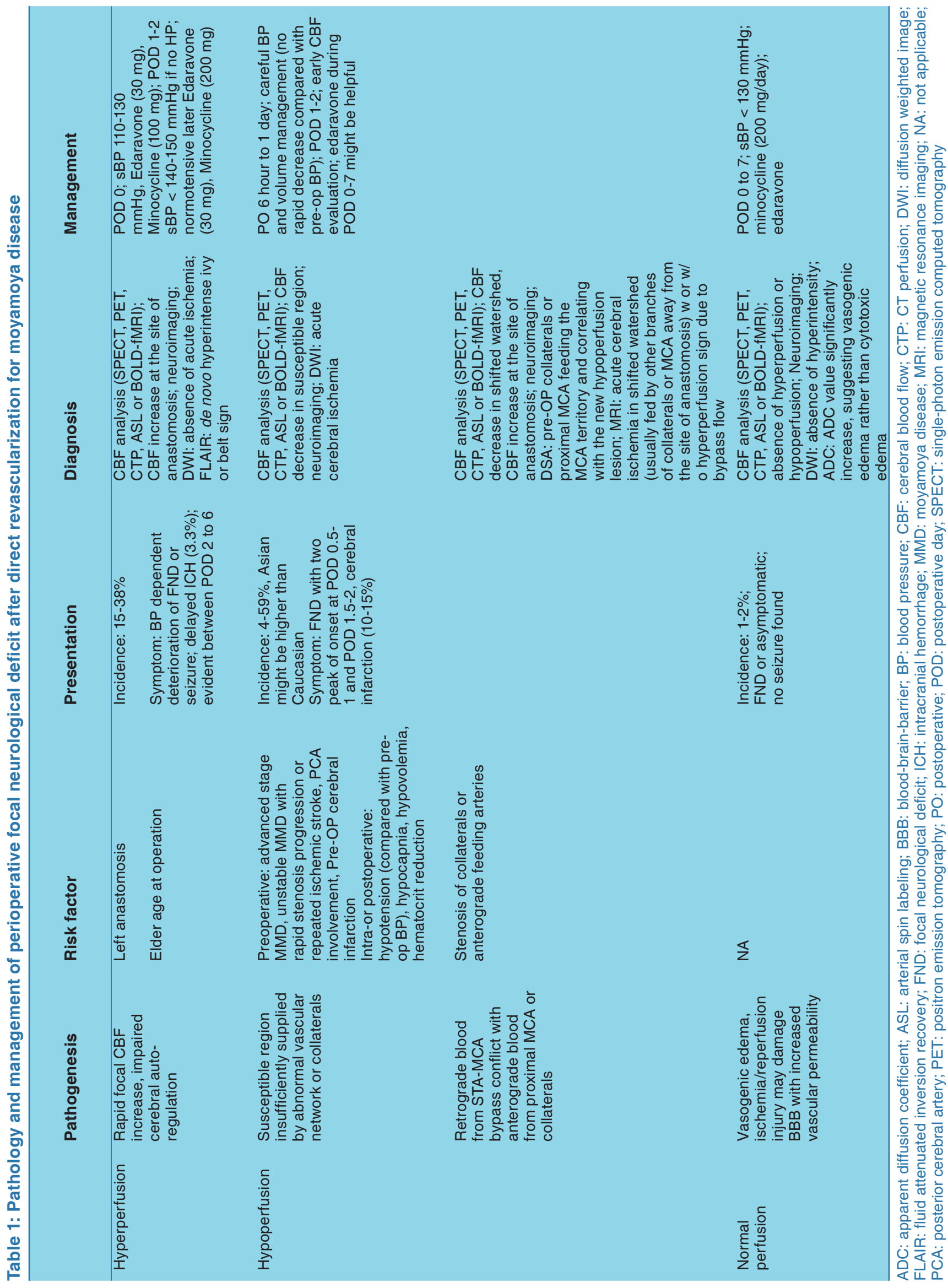



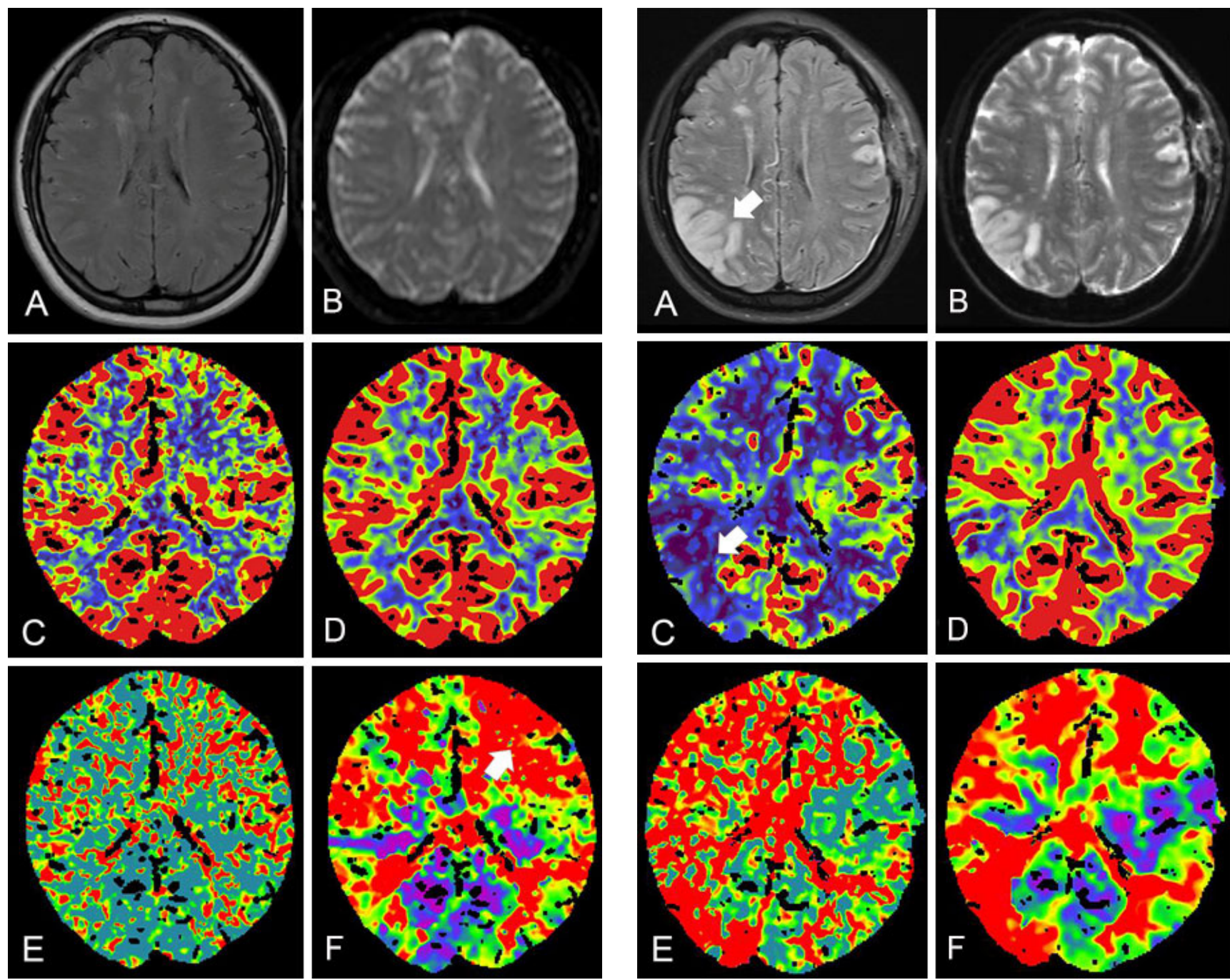

Figure 2: Preoperative magnetic resonance image and perfusion CT. No acute cerebral ischemic lesion on preoperative FLAIR (A), DWI (B), perfusion CT revealed mild reduced CBF (C), and CBV (D) along with increased MTT (E) and TTP (F) at left frontal lobe (white arrow). CT: computed tomography; CBF: cerebral blood flow; CBV: cerebral blood volume; DWI: diffusion weighted image; FLAIR: fluid attenuated inversion recovery; MTT: mean transit time; TTP: time to peak

mild blood pressure lowering might reduce the risk of hyperperfusion without increasing the incidence of ischemic stroke, ${ }^{[14]}$ although its validation still needs to be verified in other independent cohorts and high risk subgroups.

Both hyperperfusion and hypoperfusion have been identified as reasons for transient neurological deficits [Table 1]. ${ }^{[6,8,13]}$ Postoperative cerebral perfusion status might fluctuate from initial hyperperfusion after bypass surgery to local and transient hypoperfusion before it normalizes to a new level. ${ }^{[13]}$ Moreover, the perfusion at different region might also be distinct during the same period of time. ${ }^{[10]} \mathrm{CBF}$ evaluation at the early postoperative period (POD 1 to 3 ) might be recommended to tailor the perioperative management

Figure 3: Postoperative magnetic resonance image and perfusion CT. MR image at postoperative Day 6 revealed a ischemic lesion (white arrow) of hyperintensity between the right parietal and occipital lobe on FLAIR (A) and DWI (B). Perfusion CT at postoperative Day 3 showed CBF (C) and CBV (D) reduced at the corresponding site. Regional MTT (E) and TTP (F) increased significantly. CT: computed tomography; CBF: cerebral blood flow; CBV: cerebral blood volume; DWI: diffusion weighted image; FLAIR: fluid attenuated inversion recovery; MTT: mean transit time; TTP: time to peak

based on the perfusion status. ${ }^{[15]}$ The DWI and fluid attenuated inversion recovery (FLAIR) sequence could also be helpful to identify hyperperfusion and acute ischemia with typical radiological features. ${ }^{[15-17]}$ In our case, we suspected that the transient delirium might have been associated with hyperperufusion syndrome, so we followed our previous protocol with CBF analysis and neuroimaging on the third postoperative day. However, in this case, the patient's neurological event occurred within 2 days after surgery, and the imaging beyond that time did not provide a timely evaluation for tailoring management prior to the formation of the new cerebral infarction. Considering the time pattern of postoperative fluctuation in cerebral perfusion, an early 
combination of $\mathrm{CBF}$ analysis and neuroimaging within 2 days after surgery might be reasonable [Table 1]. ${ }^{[13,15]}$

Although the positron emission tomography (PET) and single-photon emission computed tomography (SPECT) are the most extensively used quantitative methods for cerebral hemodynamics assessment, the use of radionuclides and the high cost of the examination limit the serial perioperative PET or SPECT scans. Therefore, the alternative noninvasive methods without contrast agent, such as bloodoxygen-level dependent functional MRI (BOLD-fMRI) or arterial spin labeling MRI (ASL-MRI), might be of great clinical importance. Previous studies suggested that the delayed and reduced magnitude of the BOLD response might indicate the severity of compromised hemodynamics in patients with cerebral ischemic diseases. ${ }^{[18,19]}$ A most recent report observed BOLD responses to specific stimulation in patients with MMD before and after revascularization, and found a predicator for postoperative outcomes, suggesting the feasibility of using BOLD-fMRI for individualized assessment of cerebral hemodynamic impairment and hemodynamic improvement after revascularization in patients with MMD. ${ }^{[20]} \mathrm{ASL}$ is another promising technology for cerebral hemodynamics assessment in perioperative management of MMD. ${ }^{[21]}$ Several studies have demonstrated the advantage and accuracy of ASL in cerebral blood flow evaluation. ${ }^{[22,23]}$ Recent advances in multiple-parameter ASL (multiple inversion time-pulse or post labeling delay) might allow for more precise assessment of cerebral hemodynamics. ${ }^{[24-26]}$ Future studies in neuroimaging will provide better radiological tools for evaluation of cerebral blood perfusion.

Free radical scavenger and anti-inflammatory medicine might also be helpful in reducing the risk of perioperative neurological events. ${ }^{[15]} A$ recent case-control study suggested MMD patients using edaravone (a free radical scavenger) had a decreased frequency of transient neurological event after direct revascularization surgery. ${ }^{[27]}$ We added ederavone in this case as recommended in some protocols, and the neurological function markedly improved without disability, despite a cerebral infarction. Minocycline, a matrix metalloproteinase- 9 inhibitor, might also ameliorate the reperfusion injury at the site of anasotmosis and the remote area after blood pressure reduction and has been used in patients with MMD to prevent both hyperperfusion and cerebral infarction at the remote area. ${ }^{[9,28,29]}$ Further studies might be feasible to unravel the therapeutic effect of these medicines in larger populations.
Postoperative agitating delirium would increase the cerebral oxygen consumption, which might increase the risk of cerebral ischemia during the early postoperative period of MMD. We did not find any evidence opposing sedation in patients with MMD. However, most sedative agents have a potential side effect of hypotension. Although these agents might be used during anesthesia, intraoperative hypotension was common and did not independently increase the risk of postoperative ischemic complications. ${ }^{[7,30]}$ In contrast, postoperative hypotension was a significant risk factor for postoperative ischemic events. ${ }^{[7]}$ Therefore, further studies might be conducted to discuss the sedation in MMD patients with postoperative, agitating delirium.

Postoperative transient neurological events were reported to occur in $40-60 \%$ of revascularization surgery for MMD, ${ }^{[10]}$ and $4-14 \%$ of patients exhibited postoperative cerebral infarction. ${ }^{[10,31]}$ This report is congruent with previous observations that early postoperative neurological deficits usually resolve within 7 days, while irreversible cerebral infarction may persist in some patients with or without neurological deficits. ${ }^{[12]}$ The incidence of transient neurological events varies among different reports, and it seems that studies from Asian countries showed a higher rate of postoperative stroke than those from America. ${ }^{\left[{ }^{[1,32]}\right.}$ The association between perioperative neurological events and permanent neurological deficits should be further examined in future studies.

\section{Authors' contributions}

The definition of intellectual content: X.L. Chen, L. Ma Literature review: X.L. Chen, L. Ma

Manuscript preparation and editing: X.L. Chen, L. Ma, J.L. Lu

Data acquisition: Y. Chen, J.L. Lu

Concept contribution: X. Ye, R. Wang, Y.L. Zhao

Definition of intellectual content: X. Ye, R. Wang, Y.L. Zhao

Manuscript review: X. Ye, R. Wang, Y.L. Zhao

\section{Acknowledgments}

The authors would like to thank members of the Joint Cerebrovascular Surgery Study Project of Peking University International Hospital and Beijing Tiantan Hospital, and Dr. Sun Yuming of the Department of Neurosurgery at Sanbo Brain Hospital, Capital Medical University.

\section{Financial support and sponsorship}

This project was supported by the Ministry of Science and Technology of China, National Key Technology Research and Development Program (2015BAI12B04, 2013BAI09B03); Beijing Institute for Brain Disorders grant (BIBD-PXM2013_014226_07_000084); and 
National Natural Science Foundation of China (H0906 81271313 and H0906 81571110 to Y.L. Zhao and 81500995 to X.L. Chen); and China Scholarship Council (No. 201508110252 to L. Ma).

\section{Conflicts of interest}

There are no conflicts of interest.

\section{Patient consent \\ Patient consent was obtained from the patient.}

\section{Ethics approval}

The treatment and data collection in our study involving the patient is consistent with the ethical standards of the Institutional Review Board.

\section{REFERENCES}

1. Research Committee on the Pathology and Treatment of Spontaneous Occlusion of the Circle of Willis; Health Labour Sciences Research Grant for Research on Measures for Infractable Diseases. Guidelines for diagnosis and treatment of moyamoya disease (spontaneous occlusion of the circle of Willis). Neurol Med Chir 2012;52:245-66.

2. Pandey P, Steinberg GK. Neurosurgical advances in the treatment of moyamoya disease. Stroke 2011;42:3304-10.

3. Fujimura M, Tominaga T. Lessons learned from moyamoya disease: outcome of direct/indirect revascularization surgery for 150 affected hemispheres. Neurol Med Chir 2012;52:327-32.

4. Guzman R, Lee M, Achrol A, Bell-Stephens T, Kelly M, Do HM, Marks MP, Steinberg GK. Clinical outcome after 450 revascularization procedures for moyamoya disease. Clinical article. J Neurosurg 2009;111:927-35.

5. Kim SH, Choi JU, Yang KH, Kim TG, Kim DS. Risk factors for postoperative ischemic complications in patients with moyamoya disease. J Neurosurg 2005;103:433-8.

6. Ohue S, Kumon Y, Kohno K, Watanabe H, Iwata S, Ohnishi T. Postoperative temporary neurological deficits in adults with moyamoya disease. Surg Neurol 2008;69:281-6.

7. Jung YJ, Ahn JS, Kwon DH, Kwun BD. Ischemic complications occurring in the contralateral hemisphere after surgical treatment of adults with moyamoya disease. J Korean Neurosurg Soc 2011;50:492-6.

8. Fujimura M, Kaneta T, Mugikura S, Shimizu H, Tominaga T. Temporary neurologic deterioration due to cerebral hyperperfusion after superficial temporal artery-middle cerebral artery anastomosis in patients with adult-onset moyamoya disease. Surg Neurol 2007;67:273-82

9. Fujimura M, Shimizu H, Inoue T, Mugikura S, Saito A, Tominaga T. Significance of focal cerebral hyperperfusion as a cause of transient neurologic deterioration after extracranial-intracranial bypass for moyamoya disease: comparative study with non-moyamoya patients using N-isopropyl-p-[(123)I] iodoamphetamine single-photon emission computed tomography. Neurosurgery 2011;68:957-64; discussion 964-5.

10. Hayashi T, Shirane R, Fujimura M, Tominaga T. Postoperative neurological deterioration in pediatric moyamoya disease: watershed shift and hyperperfusion. J Neurosurg Pediatr 2010;6:73-81.

11. Huang AP, Liu HM, Lai DM, Yang CC, Tsai YH, Wang KC, Yang $\mathrm{SH}$, Kuo MF, Tu YK. Clinical significance of posterior circulation changes after revascularization in patients with moyamoya disease. Cerebrovasc Dis 2009;28:247-57.

12. Uchino H, Kuroda S, Hirata K, Shiga T, Houkin K, Tamaki N.
Predictors and clinical features of postoperative hyperperfusion after surgical revascularization for moyamoya disease: a serial single photon emission CT/positron emission tomography study. Stroke 2012;43:2610-6

13. Mukerji N, Cook DJ, Steinberg GK. Is local hypoperfusion the reason for transient neurological deficits after STA-MCA bypass for moyamoya disease? J Neurosurg 2015;122:90-4.

14. Fujimura M, Inoue T, Shimizu H, Saito A, Mugikura S, Tominaga T. Efficacy of prophylactic blood pressure lowering according to a standardized postoperative management protocol to prevent symptomatic cerebral hyperperfusion after direct revascularization surgery for moyamoya disease. Cerebrovasc Dis 2012;33:436-45.

15. Fujimura M, Tominaga T. Significance of cerebral blood flow analysis in the acute stage after revascularization surgery for moyamoya disease. Neurol Med Chir 2015;55:775-81.

16. Horie N, Morikawa M, Morofuji Y, Hiu T, Izumo T, Hayashi K, Nagata I. De novo ivy sign indicates postoperative hyperperfusion in moyamoya disease. Stroke 2014;45:1488-91.

17. Hamano E, Kataoka H, Morita N, Maruyama D1, Satow T1, Iihara K1, Takahashi JC. Clinical implications of the cortical hyperintensity belt sign in fluid-attenuated inversion recovery images after bypass surgery for moyamoya disease. J Neurosurg 2017;126:1-7.

18. Schaaf M, Mommertz G, Ludolph A, Geibprasert S, Mühlenbruch G, Das M, Krings T. Functional MR imaging in patients with carotid artery stenosis before and after revascularization. AJNR Am $J$ Neuroradiol 2010;31:1791-8

19. Amemiya S, Kunimatsu A, Saito N, Ohtomo K. Impaired hemodynamic response in the ischemic brain assessed with BOLD fMRI. Neuroimage 2012;61:579-90.

20. Qiao PG, Han C, Qian T, Li GJ, Yin H. BOLD-fMRI with median nerve electrical stimulation predict hemodynamic improvement after revascularization in patients with moyamoya disease. J Magn Reson Imaging 2017; doi: 10.1002/jmri.25598.

21. Hartkamp NS, van Osch MJ, Kappelle J, Bokkers RP. Arterial spin labeling magnetic resonance perfusion imaging in cerebral ischemia. Curr Opin Neurol 2014;27:42-53.

22. Goetti R, O'Gorman R, Khan N, Kellenberger CJ, Scheer I. Arteria spin labelling MRI for assessment of cerebral perfusion in children with moyamoya disease: comparison with dynamic susceptibility contrast MRI. Neuroradiology 2013;55:639-47.

23. Noguchi T, Kawashima M, Irie H, Ootsuka T, Nishihara M, Matsushima T, Kudo S. Arterial spin-labeling MR imaging in moyamoya disease compared with SPECT imaging. Eur J Radiol 2011;80:e557-62.

24. Bokkers RP, Bremmer JP, van Berckel BN, Lammertsma AA, Hendrikse J, Pluim JP, Kappelle LJ, Boellaard R, Klijn CJ. Arterial spin labeling perfusion MRI at multiple delay times: a correlative study with $\mathrm{H}(2)(15) \mathrm{O}$ positron emission tomography in patients with symptomatic carotid artery occlusion. J Cereb Blood Flow Metab 2010;30:222-9.

25. Wang R, Yu S, Alger JR, Zuo Z, Chen J, Wang R, An J, Wang B, Zhao J, Xue R, Wang DJ. Multi-delay arterial spin labeling perfusion MRI in moyamoya disease -- comparison with CT perfusion imaging. Eur Radiol 2014;24:1135-44.

26. Qiao PG, Han C, Zuo ZW, Wang YT, Pfeuffer J, Duan L, Qian T, Li GJ. Clinical assessment of cerebral hemodynamics in Moyamoya disease via multiple inversion time arterial spin labeling and dynamic susceptibility contrast-magnetic resonance imaging: a comparative study. J Neuroradiol 2017; doi: 10.1016/j.neurad.2016.12.006.

27. Uchino H, Nakayama N, Kazumata K, Kuroda S, Houkin K. Edaravone reduces hyperperfusion-related neurological deficits in adult moyamoya disease: historical control study. Stroke 2016;47:1930-2.

28. Kang HS, Kim JH, Phi JH, Kim YY, Kim JE, Wang KC, Cho BK, Kim SK. Plasma matrix metalloproteinases, cytokines and angiogenic 
factors in moyamoya disease. $J$ Neurol Neurosurg Psychiatry 2010;81:673-8.

29. Fujimura M, Niizuma $K$, Inoue $T$, Sato $K$, Endo $H$, Shimizu $H$, Tominaga T. Minocycline prevents focal neurological deterioration due to cerebral hyperperfusion after extracranial-intracranial bypass for moyamoya disease. Neurosurgery 2014;74:163-70.

30. Sakamoto T, Kawaguchi M, Kurehara K, Kitaguchi K, Furuya H, Karasawa J. Risk factors for neurologic deterioration after revascularization surgery in patients with moyamoya disease. Anesth
Analg 1997;85:1060-5.

31. Scott RM, Smith JL, Robertson RL, Madsen JR, Soriano SG, Rockoff MA. Long-term outcome in children with moyamoya syndrome after cranial revascularization by pial synangiosis. $J$ Neurosurg 2004;100:142-9.

32. Kim SK, Seol HJ, Cho BK, Hwang YS, Lee DS, Wang KC. Moyamoya disease among young patients: its aggressive clinical course and the role of active surgical treatment. Neurosurgery 2004;54:840-4. 